\title{
Safety of laparoscopic appendectomy for the management of acute appendicitis during pregnancy
}

\author{
Youn Young Park \\ Department of Surgery, Uijeongbu St. Mary's Hospital, College of Medicine, The Catholic University of Korea, Seoul, Korea
}

Laparoscopic appendectomy during pregnancy is widely accepted as a safe procedure in Korea, where the rate of laparoscopic surgery is high. Although the Society of American Gastrointestinal and Endoscopic Surgeons guideline currently mentions laparoscopic appendectomy as the treatment of choice for pregnant patients with appendicitis, some concerns arising from pneumoperitoneum during laparoscopic surgery exist in maternal postoperative and fetal outcomes. Further effort to provide firm evidence to clarify the safety of laparoscopic appendectomy during pregnancy is still needed.

Keywords: Pregnancy, Appendicitis, Laparoscopy

\author{
Received April 26, 2021 \\ Revised 1st May 6, 2021 \\ 2nd May 7, 2021 \\ Accepted May 10, 2021 \\ Corresponding author \\ Youn Young Park \\ Department of Surgery, The Catholic \\ University of Korea, Uijeongbu St. \\ Mary's Hospital, Cheonbo-ro 271, \\ Uijeongbu 11765, Korea \\ Tel: +82-31-820-5228 \\ Fax: +82-31-847-2717 \\ E-mail: yuni0225@nate.com \\ ORCID: \\ https://orcid.org/0000-0001-7724-1492
}

Copyright () The Korean Society of Endoscopic and Laparoscopic Surgeons. This is an Open Access article distributed under the terms of the Creative Commons Attribution Non-Commercial License (http://
creativecommons.org/licenses/by-nc/4.0/) which permits unrestricted non-commercial use, distribution, and reproduction in any medium, provided the original work is properly cited.

With the increase in the rate of laparoscopic surgery in Korea, laparoscopic appendectomy in pregnant women is being widely adopted by many general surgeons owing to easy access to the appendix displaced by the gravida uterus and fine visualization when compared with open appendectomy. In addition, the Society of American Gastrointestinal and Endoscopic Surgeons guideline mentions laparoscopic appendectomy as the treatment of choice for pregnant patients with appendicitis [1]. Nevertheless, performing laparoscopic appendectomy in pregnant women is still controversial owing to weak evidence and the contradictory results of recent meta-analyses and systematic reviews.

The primary issues regarding laparoscopic surgery during pregnancy are mainly addressed in two aspects. One is maternal perioperative outcomes, and the other is fetal outcomes such as fetal loss and preterm delivery. Adverse fetal outcomes might re- sult from the $\mathrm{CO}_{2}$ gas used for pneumoperitoneum, which might cause fetal acidosis, and from elevated abdominal pressure during laparoscopic surgery, which might cause decreased maternal venous return and consequently decreased fetal blood flow.

Among those controversial issues, most perioperative and fetal outcomes, except fetal loss, have been reported to be comparable to open appendectomy. The previous literature has mentioned no significant differences so far for fetal loss, except one study reported in 2007 by McGory et al. [2], which had the largest sample size (laparoscopy $[n=454]$ vs. open $[n=2,679]$; odds ratio [OR], 2.16; 95\% confidence interval, 1.41-3.29) among previous studies. Owing to this large sample size, two recent meta-analyses on this topic showed higher ORs for fetal loss after laparoscopic appendectomy compared to open appendectomy, in spite of the fact that it was the only study demonstrating worse outcome when a 
fixed effect model was adopted [3,4]. However, the authors of the two meta-analyses differently interpreted the results and concluded differently. Lee et al. [4] insisted that it is not reasonable to accept the result owing to the high weight of the study, which resulted in disproportionately high influence [4]. The other studies included in these recent meta-analyses are mostly retrospective or prospective observational studies with small sample sizes ranging from 4 to 128 in the laparoscopic appendectomy group $[3,4]$.

The article in the current issue, entitled "Safety of appendectomy during pregnancy in the totally laparoscopic age," is addressing only the postoperative outcomes of 16 pregnant women who underwent laparoscopic appendectomy for acute appendicitis treatment compared with nonpregnant women after propensity score matching [5]. As mentioned above, in this totally laparoscopic age, open appendectomy is rarely performed in Korea; therefore, the authors might not have been able to compare the outcomes with pregnant women undergoing open appendectomy during the same study period. Instead, they showed comparable postoperative outcomes, such as postoperative complications and length of stay, between the pregnant women and nonpregnant women and provided a descriptive report on fetal outcomes. In this study, the proportion of women who were in the 1st trimester was the highest, and all cases of fetal loss among patients in their 1st trimester were associated with termination of the pregnancy based on the patients' decision and not spontaneous abortion. Considering the fact that the 2nd trimester is the most common period of acute appendicitis in pregnant women and the 1st trimester is known to be related to higher fetal mortality and higher preterm delivery rates, the fetal outcomes of this study can be considered to indicate safety.

In a population-based cohort study using the National Health Insurance Research Database in Taiwan, the patients were divided into three groups according to the types of treatment (open, laparoscopic, and nonoperative treatment). When comparing the three groups to pregnant women without appendicitis, the nonoperative treatment group showed the highest ORs for abortion and preterm labor; however, no differences were found when the laparoscopic appendectomy and open appendectomy groups were compared to each other in terms of abortion and preterm labor [6]. Therefore, avoiding delay in operative management is much more important to achieve better fetal outcomes rather than the difference in surgical approach.

In summary, this study indirectly demonstrated the safety of laparoscopic appendectomy for acute appendicitis management during pregnancy. Owing to the nature of pregnant women as vulnerable study subjects, there is no room for a randomized controlled trial to assess this issue. However, further large-scale studies are needed to confirm the safety of laparoscopic appendectomy during pregnancy.

\section{NOTES}

\section{Conflict of interest}

The author has no conflict of interest to declare.

\section{REFERENCES}

1. Society of American Gastrointestinal and Endoscopic Surgeons (SAGES). Guidelines for the use of laparoscopy during pregnancy [Internet]. Los Angeles: SAGES; c2017 [cited 2021 Apr 16]. Available from: https://www.sages.org/publications/guidelines/guidelines-fordiagnosis-treatment-and-use-of-laparoscopy-for-surgical-problemsduring-pregnancy/

2. McGory ML, Zingmond DS, Tillou A, Hiatt JR, Ko CY, Cryer HM. Negative appendectomy in pregnant women is associated with a substantial risk of fetal loss. J Am Coll Surg 2007;205:534-540.

3. Frountzas M, Nikolaou C, Stergios K, Kontzoglou K, Toutouzas K, Pergialiotis V. Is the laparoscopic approach a safe choice for the management of acute appendicitis in pregnant women? A meta-analysis of observational studies. Ann R Coll Surg Engl 2019;101:235-248.

4. Lee SH, Lee JY, Choi YY, Lee JG. Laparoscopic appendectomy versus open appendectomy for suspected appendicitis during pregnancy: a systematic review and updated meta-analysis. BMC Surg 2019;19:41.

5. Seok JW, Son J, Jung KU, Lee SR, Kim HO. Safety of appendectomy during pregnancy in the totally laparoscopic age. J Minim Invasive Surg 2021;24:68-75.

6. Cheng HT, Wang YC, Lo HC, et al. Laparoscopic appendectomy versus open appendectomy in pregnancy: a population-based analysis of maternal outcome. Surg Endosc 2015;29:1394-1399. 